\title{
Obesity and the microbiome - a surgeon's perspective
}

\begin{abstract}
Obesity is an enormous public health problem, arising from the abundance of food, eating behavior, and body regulation on energy intake, expenditure, and storage. Recent evidence suggests that the gut microbiota may play a role in obesity by increasing the host's energy-harvesting efficiency. Obesity surgery, a well-accepted tool for weight reduction, contributes to alterations in gut microbiota through anatomical changes and through modifications of dietary components. The mechanisms of the microbiome activity consist of modulating the host metabolism, inflammatory processes including insulin resistance, and by affecting the endocrine and the nervous systems.
\end{abstract}

The type of bacteria present in the gut regulates fatty acid metabolism by using specific short chain fatty acids as metabolites. Bacterial fermentation of dietary fibers producing these fatty acids turns the dietary fibers into mediators for bacterial communication with the organs, thereby modulating the host metabolism. Bariatric surgery changes the ratio of microbiome components. Studies suggest that there is not only a starvation-like adaptation of the gut microbiota but also a change in the relative abundance of different bacteria that are associated with metabolic and inflammatory processes. These changes were found at three months post-surgery, and are persistent at longer follow-ups, indicating a stable gut modification.

The literature review presented may suggest that specific analysis of the microbiota can help in selecting the preferred type of surgery and the prospect of surgery success in terms of long term weight reduction. We thought it would be beneficial for scientists interested in obesity to enrich their knowledge about the highlights of the relations between the microbiota and obesity, and more specifically with that of bariatric surgery.

Keywords: microbiome, obesity, bariatric surgery, gut microbiota
Volume 7 Issue 3 - 2017

\author{
Asnat Raziel,' Nasser Sakran, ',2 David \\ Goitein ${ }^{1,3}$ \\ 'Assia Medical Group, Israel \\ ${ }^{2}$ Emek Medical Center, Technion Israel Institute of Technology, \\ Israel \\ ${ }^{3}$ Department of Surgery C, Tel Aviv University, Israel
}

Correspondence: Asnat Raziel,Assia Medical Group, Assuta Medical Center, 20 Habarzel Street, Tel Aviv, 69710, Israel, Tel +97237645444, Fax +97237644445, Email drraziel@zahav.net.il

Received: August 19, 2017| Published: September 14, 2017
Abbreviations: BAC, bacteroides; PREV, prevotella; RUM, ruminococcus; ANGPTL4, angiopoietin-like protein 4; SCFAs, short-chain fatty acids; TNF- $\alpha$, tumor necrosis factor- $\alpha$; T2D, type 2 diabetes; GLP-1, glucagon-like peptide 1; GLP-2, glucagon-like peptide 2; PYY, peptide yy; RYGB, roux-en-y gastric bypass

\section{Introduction}

Obesity is the result of an imbalance between energy intake and expenditure. Some genetic factors contribute to the control and maintenance of body weight, but the dramatic increase in obesity prevalence over the past decades, suggests an additional, environmental effect. ${ }^{1}$ The effect of the gut microbiome in controlling obesity phenotypes ${ }^{2}$ has generated attention to its role in developing obesity. In experimental germ-free mice models, when the luminal contents from the ceca of obese or lean mice were implanted, mice receiving the microbiome of obese donors gained more weight than recipients of the lean donors, despite equivalent food intake. ${ }^{3}$ Germ free mice that had fecal transplant from normal mice, got fat in spite of the reduced food intake. ${ }^{4}$

The microbiome combines both genetic and environmental components making it de facto an extension of the genome. ${ }^{5}$ The bacterial components of the microbiome have been intensively studied in recent years, driven by large-scale projects such as the Human Microbiome Project. ${ }^{6}$ These studies have established the existence of substantial variability in the bacterial composition of healthy individuals, with identical twins sharing less than $50 \%$ of their bacterial mass. The mapping of the human genome as well as the human microbiome focused the attention of scientists involved in the fight against obesity to look for a possible solution in hereditary factors rather than on healthy lifestyle. With evidence describing major changes in gut microbiota following bariatric surgery ${ }^{7-11}$ we believe it is important to summarize the myriad of data on the link between the microbiota and various metabolic systems, and to present the possible relationship between the microbiota and obesity, and more explicitly with bariatric surgery.

\section{Methods}

\section{Data sources}

A broad search of the English-language literature was performed. The electronic search included MEDLINE and Google Scholar, with cutoff of publications from 2010. Early pioneering articles were included for the sake of completeness of the review. For simplification of the review, discussions on the relationship of the microbiota with human metabolism were filtered to exclude animal data (unless absolutely necessary) and changes that were still under debate. Specific, surgery-related terms such as bariatric surgery and microbiome /microbiota were explored separately in the same electronic data bases. Full articles were obtained for all articles and for any citations for which abstract data was found relevant. 


\section{Discussion}

\section{Mechanism of microbiota action}

The composition of the microbiota including bacteria, viruses, and eukaryotes is relatively stable over time in healthy adult individuals. However, this temporal consistency only exists when variables such as diet, disease-state, and environment, are constant. Dietary changes, in particular, have significant effects on the microbiota. Shifting from a high-fat/low-fiber diet to a low-fat/high-fiber diet in human volunteers causes notable changes in the gut microbiota within 24 hours. ${ }^{6}$ Bacteria, typically members of the Bacteroidetes and Firmicutes divisions, dominate the gut microbiota at least in volume with much variability in the gut's bacterial mass. The microbiota of most individuals can be categorized into one of three variants or "enterotypes" based on the dominant classes: Bacteroides (BAC), Prevotella (PREV), or Ruminococcus (RUM). Each of these variants is characterized by a ratio of the abundance of the sum BAC+RUM relative to PREV. These broad patterns are driven primarily by dietary effects. $^{6}$

In animal obesity models, the interplay between the dominant gut phyla, Bacteroidetes and Firmicutes, is shifted with a significant reduction of the former and a corresponding increase in the latter. ${ }^{12}$ The increased ratio of Firmicutes to Bacteroidetes in $o b / o b$ mice promote adiposity and represents a host-mediated adaptive response to limited energy uptake and storage. ${ }^{12}$ A greater representation of Firmicutes and fewer Bacteroidetes characterizes obese host microbiota. In humans, obesity is associated with a depletion of Bacteroidetes as well, but alterations in the microbiota associated with weight-reduction diets and weight loss vary between studies. ${ }^{13}$ The variation in data depends on several factors: in humans, it is hard to control the type of food consumed during diet, in contrast to the specific formulae given to animals. In addition, methods for elucidation of the microbiome composition vary between studies and may lead to different results. ${ }^{13}$

\section{Host metabolism}

The gut microbiota regulates angiopoietin-like protein 4 (ANGPTL4), an important regulator of host lipid metabolism. ANGPTL4 regulates fatty acid oxidation in both muscle and adipose tissue. ${ }^{14}$ When a normal mouse microbiota is administered to germfree mice, ANGPTL4 production is suppressed in the intestine and a greater proportion of triglycerides are deposited in adipose tissue. The relevance of these findings to human health is complex because of the restrictions of genetic studies in humans. Nevertheless, variants of the ANGPTL4 gene were found to be more prevalent in individuals with comparatively low triglyceride levels. ${ }^{15}$

Moreover, gut bacteria can produce substrate-dependent specific metabolites such as short-chain fatty acids (SCFAs) that are present in the gut lumen. The relative abundance of SCFAs and their relative ratios lead to specific host responses. The type of bacteria present in the gut regulates this aspect of fatty acid metabolism as specific bacteria use particular SCFAs as metabolites. ${ }^{16}$ Bacterial fermentation of dietary fibers in the intestine is a major source of SCFAs, making dietary fibers key players in mediating bacteria communication with the organs, thereby modulating host metabolism.

\section{Insulin resistance and inflammation}

Obesity and metabolic syndrome are associated with low-grade metabolic inflammation. An increase in certain cytokines (e.g. tumor necrosis factor- $\alpha(\mathrm{TNF}-\alpha))$, promotes insulin resistance. A highfat diet results in incorporation of triglycerides into chylomicrons, which also have a high affinity for lipopolysaccharides, thus increasing lipopolysaccharide absorption and triggering metabolic inflammation. ${ }^{17}$ In animal models, subcutaneous infusion of lipopolysaccharides resulted in weight gain, insulin resistance and induction of an inflammatory state, even without diet modification..$^{18} \mathrm{In}$ a similar model, a high fat diet was shown to modify the proportion of BAC-related bacteria and reduce that of Bifidobacteria. ${ }^{13}$ Higher levels of Bifidobacteria in the gut, achieved directly as an ingested probiotic or indirectly with bifidogenic prebiotics, reduced inflammation and improved glucose tolerance. ${ }^{13}$ Another study found that abundant Bifidobacteria decreased the amount of lipopolysaccharides diffusing to the plasma. ${ }^{19}$ In humans, a correlation was found between plasma lipopolysaccharides levels, the energy produced from diet, and type 2 diabetes (T2D). ${ }^{18}$ Both Mediterranean and a high complexcarbohydrate diet produced changes in the gut microbiota and caused a protective effect for T2D. ${ }^{20}$

\section{The microbiome and the endocrine system}

Endocrine signals coordinate energy intake and expenditure from the gut to the brain. The gut signals nutrient intake by secreting incretins (e.g. glucagon-like peptides 1 and 2 (GLP-1 and GLP-2)). GLP-1 stimulates insulin release, promotes satiety and slows gastric emptying, thus promoting weight loss..$^{21}$ GLP-2 stimulates intestinal glucose transport and reduces gut permeability. ${ }^{21}$ Studies in mice and rats have shown a connection between gut microbiota, GLP-1 and GLP-2. Genetically obese mice treated with prebiotic carbohydrates showed increased levels of GLP-1 and GLP-2 and an altered mass of gut microbiota, ${ }^{19}$ confirming a direct link between the endocrine system and gut microbiota. In other studies, microbial fermentation of oligofructose for two weeks significantly increased satiety, reduced hunger, and reduced the desire to ingest food..$^{22}$ Reduced hunger and increased satiety were also linked with changes in plasma GLP1 and Peptide YY (PYY) levels ${ }^{23}$ and with a reduced concentration of ghrelin in obese patients. ${ }^{24}$ Several non-digestible carbohydrates modulate bacterial activity while gut microbes are able to transform specific amino acids into substances that can change the secretion of GLP-1. ${ }^{16}$

\section{The microbiome and the nervous system}

The clinical link between the gut function and the nervous system can be demonstrated by common phenomena, such as diarrhea and constipation that are affected by stress and by the extensive innervation of the gut. Preclinical data suggests that intestinal microbiota play an important role in bidirectional signaling between the gut and the nervous system. For example, in irritable bowel disease (IBS), pathogenic bacteria in the gut and the nervous system are coupled..$^{25}$ Regular intake of certain probiotic bacteria can help in treating the symptoms of IBS, such as bloating and visible abdominal distention. Additionally, altered bowel habits, are also linked to plasma levels of systemic stress mediators that control the activity of the nervous system. This subject has been carefully reviewed by Rhee et al. ${ }^{26}$

\section{Effect of bariatric surgery on gut microbiota}

One of the major benefits of bariatric surgery, beyond weight loss, is the favorable metabolic change, mainly the remission of T2D. The mechanism at play is multifactorial ${ }^{27}$ but changes in gut microbiota appear to play an important role.

Roux-en-Y gastric bypass (RYGB) involves significant changes to the gut anatomy leading several groups to look into the resulting changes in the gut microbiome. A recent animal study compared rats after duodenal-jejunal bypass to controls with sham surgery or to rats 
receiving daily injection of a GLP-1 agonist. The rats that underwent surgery showed decreased concentrations of Bacteroidia and an increased abundance of Gamma-proteobacteria, compared to controls and to GLP-1-injected mice. ${ }^{9}$ Deciphering the mechanisms behind such changes is not trivial. In addition to the anatomical changes, nutritional changes might influence the microbiome as well. Zhang and co-workers showed a decrease in Firmicutes and a major elevation in Gammaproteobacteria after RYGB. ${ }^{11}$ They also detected significantly higher numbers of hydrogen utilizing methanogenic Archaea in obese individuals compared to normal-weight and post-RYBG patients. The high abundance of hydrogen producing bacteria in obese individuals has led to the hypothesis that interspecies hydrogen transfer between bacterial and archaeal species is an important mechanism for increasing energy uptake by the human large intestine. The significant shift in bacterial population after surgery may reflect a dual effect on the gut, instigated by the surgical procedure and consequently changed by food ingestion and digestion. Components of the gut microbiota of obese patients undergo a quick adaptation process as a result of $\mathrm{RYGB}^{7}$ with the BAC/PREV ratio initially lower in obese subjects, increasing at three months post RYGB.

Escherichia coli species also increase at three months postsurgery and are inversely correlated with fat mass and leptin levels, independent of changes in food intake. The Lactobacillus/ Leuconostoc/ Pediococcus group (lactic acid bacteria) and the Bifidobacterium genus all decrease after surgery. In addition, Faecalibacterium prausnitzii species are lower in subjects with diabetes and have a negative correlation with inflammatory markers before and throughout follow-up, independent of food intake. These results suggest that there is not only a starvation-like adaptation of the gut microbiota but also that the F. prausnitzii species are directly associated with a reduced low-grade inflammation state in obesity and diabetes independent of caloric intake. ${ }^{?}$

A recent study ${ }^{10}$ has compared changes in gut microbiome following both RYGB and sleeve gastrectomy (SG). At baseline (following a two-week weight reduction diet), no significant differences in gut microbiota composition was found. However, baseline gut microbiota among subjects who obtained T2D remission post-surgery showed greater Actinobacteria (phylum and class) levels, while those for which T2D did not improve showed greater Desulfovibrio levels.

Functionally, subsequent T2D remission was linked with inosine

Table I Summary of basic findings monophosphate components and higher biosynthesis of unsaturated fatty acids. After RYGB, there were three major bacteria population changes, with increases in Firmicutes and Actinobacteria and a decrease in Bacteroidetes. After SG, the Bacteroidetes population increased, which is in contrast with other studies. ${ }^{7}$ These changes may be attributed to the very low calorie diet prescribed to all participants before baseline, which is similar to the calorie intake post-surgery. Another factor that might have influenced the results is the prescription of proton-pump inhibitors post-surgery. This study stopped protonpump inhibitors three months after surgery, increasing the acidity of the gut thus promoting growth of Bacteroidetes.

Some insight on the possible mechanisms involved in post-surgery changes can be obtained by a recent animal study. ${ }^{8}$ Diet-induced obese mice had SG or sham surgery (controls). They were housed individually or cohoused with control mice of the same weight. Weight changes and insulin resistance post SG persisted despite continued exposure to high fat diet, as well as ingestion of feces of weightmatched, controls. Moreover, cohoused controls showed a shift in microbial community composition and had significantly different composition than did individually-housed controls $(\mathrm{P}=0.026)$. Conversely, microbial communities from individually housed $\mathrm{SG}$ mice did not differ from microbial communities from cohoused SG mice $(\mathrm{P}=0.214)$. Thus, when exposed to the feces of SG mice, the composition of controls changed, while surgically-induced changes in microbiota were irreversible.

\section{Conclusion}

Recent work has explored the importance of the intestinal microbiome on metabolism. The microbiome has been shown to alter susceptibility to both obesity and T2D through mechanism involving the metabolic, endocrine and nervous systems. Obesity itself has also been found to alter the microbiome by reducing microbial diversity and bacterial genes.

Bariatric surgery has a combined effect on the gut microbiome. It manipulates the gut environment and also causes weight loss. It therefore has the potential to influence the balance of bacterial composition and abundance within the intestinal system, either directly, through environmental changes or indirectly, through weight loss. More studies, both clinical and preclinical are needed to shed more light on this important topic. The basic findings described in this review are summarized in Table 1.

Mechanism of microbiota action

\begin{tabular}{ll}
\hline Metabolic path & Essential mechanism \\
\hline Host Metabolism & Regulation of ANGPTL4 production \\
& Production of substrate-dependent specific metabolites such as SCFAs $\quad$ I6
\end{tabular}

Insulin Resistance and Inflammation

Abundant Bifidobacteria decreased the amount of lipopolysaccharides diffusing to the plasma affecting energy produced from diet and T2D

Higher levels of Bifidobacteria in the gut, achieved through ingested probiotic or bifidogenic prebiotics, improved glucose tolerance.

The gut signals nutrient intake by secreting GLP-I and GLP-2. GLP- I stimulates insulin release, promotes satiety and slows gastric emptying. GLP-2 stimulates intestinal glucose transport and reduces gut permeability.

The microbiome and the endocrine system 
Table Continued....

\begin{tabular}{ll}
\hline Mechanism of microbiota action & Essential mechanism \\
\hline Metabolic path & $\begin{array}{l}\text { Pathogenic bacteria in the gut in IBS, couple the nervous system to gut } \\
\text { bacteria }\end{array}$ \\
\hline The microbiome and the nervous system & $\begin{array}{l}\text { Changes in bowel habits are linked levels of systemic stress mediators in } \\
\text { the plasma that control and thus the activity of the nervous system. }\end{array}$ \\
\hline
\end{tabular}

\section{Effect of bariatric surgery on gut microbiota}

Obese individuals produce significantly higher numbers of hydrogen utilizing methanogenic Archaea compared to normal-weight and post-RYBG patients. This high abundance suggests that interspecies hydrogen transfer between bacterial and archaeal species is an important mechanism for increasing energy uptake by the human large intestine.

Escherichia coli species that are inversely correlated with fat mass and leptin levels increase at three months postsurgery, independent of changes in food intake

When comparing changes in gut microbiome following RYGB and SG, the post-surgery T2D remission was linked to the levels of bacteria following a pre-surgery two-week weight reduction diet.

When exposed to the feces of mice that had SG, the composition gut bacteria of sham-surgery mice changed, while surgically-induced changes in microbiota were irreversible, independent of co-housing with sham-surgery mice.

\section{Acknowledgements}

None.

\section{Conflict of interest}

The author declares no conflict of interest.

\section{References}

1. Khan MJ, Gerasimidis K, Edwards CA, et al. Role of gut microbiota in the aetiology of obesity: proposed mechanisms and review of the literature. J Obes. 2016;2016:7353642.

2. Le Chatelier E, Nielsen T, Qin J, et al. Richness of human gut microbiome correlates with metabolic markers. Nature. 2013;500:541-546.

3. Turnbaugh PJ, Ley RE, Mahowald MA, et al. An obesity-associated gut microbiome with increased capacity for energy harvest. Nature. 2006;444(122):1027-1131.

4. Bäckhed F, Ding $\mathrm{H}$, Wang $\mathrm{T}$, et al. The gut microbiota as an environmental factor that regulates fat storage. Proc Natl Acad Sci USA. 2004;101(44):15718-15723.

5. Turnbaugh PJ, Ley RE, Hamady M, et al. The human microbiome project: exploring the microbial part of ourselves in a changing world. Nature. 2007;449(7164):804810.

6. Clemente JC, Ursell LK, Parfrey LW, et al. The impact of the gut microbiota on human health: an integrative view. Cell. 2012;148(6):1258-1270.

7. Furet JP, Kong LC, Tap J, et al. Differential adaptation of human gut microbiota to bariatric surgery-induced weight loss. Diabetes. 2010;59(12):3049-3057.

8. Jahansouz C, Staley C, Bernlohr DA, et al. Sleeve gastrectomy drives persistent shifts in the gut microbiome. Surg Obes Relat Dis. 2017;13(6):916-924.

9. Kashihara H, Shimada M, Yoshikawa K, et al. Duodenal-jejunal bypass changes the composition of the gut microbiota. Surg Today. 2017;47(1):137-140.
10. Murphy R, Tsai P, Jüllig M, et al. Differential changes in gut microbiota after gastric bypass and sleeve gastrectomy bariatric surgery vary according to diabetes remission. Obes Surg. 2016;27(4):917-925.

11. Zhang H, DiBaise JK, Zuccolo A, et al. Human gut microbiota in obesity and after gastric bypass. Proc Natl Acad Sci USA. 2009;106(7):23652370 .

12. Ley RE, Bäckhed F, Turnbaugh $\mathrm{P}$, et al. Obesity alters gut microbial ecology. Proc Natl Acad Sci USA. 2005;102(31):11070-11075.

13. Ley RE. Obesity and the human microbiome. Curr Opin Gastroenterol. 2010;26(1):5-11.

14. Mandard S, Zandbergen F, van Straten E, et al. The fasting-induced adipose factor/angiopoietin-like protein 4 is physically associated with lipoproteins and governs plasma lipid levels and adiposity. J Biol Chem. 2006;281(2):934-944.

15. Romeo S, Pennacchio LA, Fu Y, et al. Population-based resequencing of ANGPTL4 uncovers variations that reduce triglycerides and increase HDL. Nat Genet. 2007;39(4):513-516.

16. Cani PD, Knauf C. How gut microbes talk to organs: The role of endocrine and nervous routes. Mol Metab. 2016;5:743-752.

17. Ghoshal S, Witta J, Zhong J, et al. Chylomicrons promote intestinal absorption of lipopolysaccharides. J Lipid Res. 2009;50(1):90-97.

18. Creely SJ, Mc Ternan PG, Kusminski CM, et al. Lipopolysaccharide activates an innate immune system response in human adipose tissue in obesity and type 2 diabetes. Am J Physiol Endocrin Metab. 2007;292(3):740E-747E.

19. Cani PD, Possemiers S, Van de Wiele T, et al. Changes in gut microbiota control inflammation in obese mice through a mechanism involving GLP-2-driven improvement of gut permeability. Gut. 2009;58(8):10911103.

20. Haro C, Montes-Borrego M, Rangel-Zuniga OA, et al. Two healthy diets modulate gut microbial community improving insulin sensitivity in a human obese population. Am $J$ Physiol Endocrinol Metab. 2016;101(1):233-242. 
21. Drucker DJ. Gut adaptation and the glucagon-like peptides. Gut 2002;50(3):428-435.

22. Cani PD, Joly E, Horsmans Y, et al. promotes satiety in healthy human: a pilot study. Eur J Clin Nutr. 2006;60(5):567-572.

23. Cani PD, Lecourt E, Dewulf EM, et al. Gut microbiota fermentation of prebiotics increases satietogenic and incretin gut peptide production with consequences for appetite sensation and glucose response after a meal. Am J Clin Nutr. 2009;90(5):1236-1243.

24. Parnell JA, Reimer RA. Weight loss during oligofructose supplementation is associated with decreased ghrelin and increased peptide YY in overweight and obese adults. Am J Clin Nutr. 2009;89(6):1751-1759.
25. Collins SM, Bercik P. The relationship between intestinal microbiota and the central nervous system in normal gastrointestinal function and disease. Gastroenterology. 2009;136(6):2003-2014.

26. Rhee SH, Pothoulakis C, Mayer EA. Principles and clinical implications of the brain-gut-enteric microbiota axis. Nat Rev Gastroenterol Hepato. $2009 ; 6(5): 306-314$.

27. Yamamoto H, Kaida S, Yamaguchi T, et al. Potential mechanisms mediating improved glycemic control after bariatric/metabolic surgery. Surg Today. 2016;46(3):268-274. 